\title{
Conventional and fuzzy force control in robotised machining
} HENDZEL Zenon ${ }^{1, a}$, BURGHARDT Andrzej ${ }^{1, b}$, GIERLAK Piotr ${ }^{1, c}$ and SZUSTER Marcin ${ }^{1, d}$

\author{
${ }^{1}$ Department of Applied Mechanics and Robotics, Rzeszow University of Technology, \\ 8 Powstańców Warszawy St., 35-959 Rzeszów, Poland \\ azenhen@prz.edu.pl, ${ }^{b} a n d r z e j b @ p r z . e d u . p l,{ }^{c}$ pgierlak@prz.edu.pl, ${ }^{d}$ mszuster@prz.edu.pl
}

Keywords: manipulator control, force control, PID control, fuzzy control, robotised machining.

\begin{abstract}
This article presents an application of the hybrid position-force control of the robotic manipulator with use of artificial neural networks and fuzzy logic systems in complex control system. The mathematical description of the manipulator and a closed-loop system are presented. In the position control were used the PD controller and artificial neural networks, which compensate nonlinearities of the manipulator. The paper presents mainly the application of various strategies of the force control. The force control strategies using conventional controllers P, PI, PD, PID and fuzzy controllers are presented and discussed. All of the control methods were verified on the real object in order to make a comparison of a control quality.
\end{abstract}

\section{Introduction}

Robotic manipulators are devices which find different applications in many domains of the economy. During the robotised machining process the tip of the manipulator comes into contact with the environment, what results in occurrence of interaction forces. In contemporary industrial applications it is desired for the manipulator to exert specified forces and move along a prescribed path. Therefore the hybrid position-force control should be applied. The problem of the manipulator hybrid position-force control $[1,2,3,4,5,6]$ is complex, because the manipulator is a nonlinear object, whose parameters may be unknown, variable and the working conditions are changeable. The hybrid control consists of a position control, which realises movement in the so-called contact surface, and a force control, which realises an interaction force normal to the surface. In the position control the PD and neural controller [7] are applied. Application of adaptive artificial neural networks (ANNs) in the control system assures of correct tracking control of the manipulator even in a case, when the mathematical model of the control object is unknown. In this work, the hybrid position-force controller with a neural compensation of the manipulator nonlinearities is applied [3].

Presented in the paper hybrid control algorithms will be applied to control of a robotised machining. The force control task is very important due to the fact that the downforce is one of the machining process parameters. In the presented work many strategies of the force control have been tested. Besides the conventional controllers were proposed their fuzzy equivalents and two versions of the nonlinear fuzzy controller based on PI [8], that provided significant increase of the force control quality, what is very important in parts machining, e.g. edging.

\section{The description of the manipulator's mathematical model and the closed loop system}

Dynamical equations of motion of the analysed manipulator are in the following form $[1,2]$

$$
\mathbf{M}(\mathbf{q}) \ddot{\mathbf{q}}+\mathbf{C}(\mathbf{q}, \dot{\mathbf{q}}) \dot{\mathbf{q}}+\mathbf{F}(\dot{\mathbf{q}})+\mathbf{G}(\mathbf{q})+\boldsymbol{\tau}_{\mathrm{d}}(\mathrm{t})=\mathbf{u}+\mathbf{J}_{\mathrm{h}}^{\mathrm{T}}(\mathbf{q}) \lambda+\mathbf{J}^{\mathrm{bT}}(\mathbf{q}) \mathbf{F}_{\mathrm{E}}
$$

where, $\mathbf{q} \in \mathrm{R}^{\mathrm{n}}$ - the vector of generalised coordinates, $\mathbf{M}(\mathbf{q}) \in \mathrm{R}^{\mathrm{n} \times \mathrm{n}}$ - the inertia matrix, $\mathbf{C}(\mathbf{q}, \dot{\mathbf{q}}) \dot{\mathbf{q}} \in \mathrm{R}^{\mathrm{n}}$ - the vector of centrifugal and Coriolis forces/moments, $\mathbf{F}(\dot{\mathbf{q}}) \in \mathrm{R}^{\mathrm{n}}$ - the friction vector, $\mathbf{G}(\mathbf{q}) \in \mathrm{R}^{\mathrm{n}}$ - the gravity vector, $\boldsymbol{\tau}_{\mathrm{d}}(\mathrm{t}) \in \mathrm{R}^{\mathrm{n}}$ - the vector of disturbances bounded by $\left\|\boldsymbol{\tau}_{\mathrm{d}}\right\|<\mathrm{b}$, 
$\mathrm{b}>0, \quad \mathbf{u} \in \mathrm{R}^{\mathrm{n}}$ - the control input vector, $\mathbf{J}_{\mathrm{h}}(\mathbf{q}) \in \mathrm{R}^{\mathrm{ml} \times \mathrm{n}}$ - a Jacobian matrix associated with the contact surface geometry, $\lambda \in \mathrm{R}^{\mathrm{m} 1}$ - a vector of constraining forces exerted normally to the contact surface (Lagrange multiplier), $\mathbf{J}^{\mathrm{bT}}(\mathbf{q}) \mathbf{F}_{\mathrm{E}}$ - a vector of forces/moments in joints, which come from forces/moments $\mathbf{F}_{\mathrm{E}} \in \mathrm{R}^{\mathrm{m}}$ applied to the end-effector (except the constraining forces). The vector $\mathbf{F}_{\mathrm{E}}$ can include e.g. friction forces, which are tangential to the contact surface. The matrix $\mathbf{J}^{\mathrm{b}}(\mathbf{q}) \in \mathrm{R}^{\mathrm{m} \times \mathrm{n}}$ is a geometric jacobian in body [9]. In this description $\mathrm{n}, \mathrm{m}$ and $\mathrm{m} 1$ denote respectively: the number of degrees of freedom, the size of the workspace, the number of directions, in which motion limits exist. The jacobian matrix $\mathbf{J}_{\mathrm{h}}$ arises from an equation of the holonomic constraints, which describes the contact surface, and has the following form

$$
\mathbf{J}_{\mathrm{h}}(\mathbf{q})=\frac{\partial \mathbf{h}(\mathbf{q})}{\partial \mathbf{q}},
$$

where $\mathbf{h}(\mathbf{q})=\mathbf{0}$ - the equation of constraints. Due to the holonomic constraints the number of degrees of freedom is reduced to $\mathrm{n} 1=\mathrm{n}-\mathrm{m} 1$. Thus vector $\mathbf{q}$ can be decomposed as follows $\mathbf{q}^{\mathrm{T}}=\left[\boldsymbol{\theta}_{1}{ }^{\mathrm{T}} \boldsymbol{\theta}_{2}{ }^{\mathrm{T}}\right]^{\mathrm{T}}$, where $\boldsymbol{\theta}_{1}$ denotes the vector of reduced position variable, and the vector $\boldsymbol{\theta}_{2}$ depends on $\boldsymbol{\theta}_{1}$ in the following way $\boldsymbol{\theta}_{2}=\boldsymbol{\gamma}\left(\boldsymbol{\theta}_{1}\right)$. The details of the reduced dynamics can be found in the works [1,3]. Let us to define the following variables

$$
\mathbf{e}_{\theta}=\boldsymbol{\theta}_{1 \mathrm{~d}}-\boldsymbol{\theta}_{1}, \quad \mathbf{s}=\dot{\mathbf{e}}_{\theta}+\Lambda \mathbf{e}_{\theta}, \quad \tilde{\lambda}=\lambda_{\mathrm{d}}-\boldsymbol{\lambda}, \quad \mathbf{v}_{1}=\dot{\boldsymbol{\theta}}_{1 \mathrm{~d}}+\Lambda \mathbf{e}_{\theta},
$$

where $\boldsymbol{\theta}_{1 \mathrm{~d}}=\boldsymbol{\theta}_{1 \mathrm{~d}}(\mathrm{t})$ - the desired motion trajectory in the reduced position variable, $\mathbf{e}_{\theta}$ - the motion error, $\mathbf{s}$ - the filtered motion error, $\boldsymbol{\Lambda}$ - the positive definite diagonal design matrix, $\lambda_{d}=\lambda_{d}(t)-$ the desired force trajectory, $\tilde{\lambda}$ - the force error, $\boldsymbol{v}_{1}-$ the auxiliary variable. This allows to describe the system in terms of the filtered motion error $\mathbf{s}$ in the form

$$
\overline{\mathbf{M}} \dot{\mathbf{s}}=-\overline{\mathbf{V}}_{1} \mathbf{s}+\mathbf{L}^{\mathrm{T}}\left[\mathbf{f}\left(\boldsymbol{\theta}_{1}, \dot{\boldsymbol{\theta}}_{1}, \mathbf{v}_{1}, \dot{\mathbf{v}}_{1}\right)+\boldsymbol{\tau}_{\mathrm{d}}-\mathbf{J}^{\mathrm{bT}} \mathbf{F}_{\mathrm{E}}-\mathbf{u}\right]
$$

where $\overline{\mathbf{M}}=\mathbf{L}^{\mathrm{T}} \mathbf{M L}, \overline{\mathbf{V}}_{1}=\mathbf{L}^{\mathrm{T}}(\mathbf{M} \dot{\mathbf{L}}+\mathbf{C L}), \mathbf{f}\left(\boldsymbol{\theta}_{1}, \dot{\boldsymbol{\theta}}_{1}, \mathbf{v}_{1}, \dot{\mathbf{v}}_{1}\right)$ - the nonlinear function, which depends on manipulator dynamics, $\mathbf{L}$ - the extended jacobian, which involves the velocity $\dot{\mathbf{q}}$ and $\dot{\boldsymbol{\theta}}_{1}$. Its form depends on the equation of constraint

$$
\mathbf{L}\left(\boldsymbol{\theta}_{1}\right)=\left[\begin{array}{l}
\mathbf{I}_{\mathrm{n} \times \mathrm{n} 1} \\
\frac{\partial \boldsymbol{\gamma}\left(\boldsymbol{\theta}_{1}\right)}{\partial \boldsymbol{\theta}_{1}}
\end{array}\right],
$$

where $\mathbf{I}_{\mathrm{n} 1 \mathrm{xn} 1}$ - the identity matrix. According to the theory of nonlinear system control, the structure of hybrid position-force control signal was assumed in the following form

$$
\mathbf{u}=\mathbf{u}_{\mathrm{C}}+\mathbf{u}_{\mathrm{PD}}-\mathbf{u}_{\mathrm{F}}-\mathbf{v}=\hat{\mathbf{f}}\left(\boldsymbol{\theta}_{1}, \dot{\boldsymbol{\theta}}_{1}, \mathbf{v}_{1}, \dot{\mathbf{v}}_{1}\right)+\mathbf{K}_{\mathrm{D}} \mathbf{L s}-\mathbf{J}_{\mathrm{h}}^{\mathrm{T}}\left[\lambda_{\mathrm{d}}+\boldsymbol{\sigma}_{\mathrm{F}}\right]-\mathbf{J}^{\mathrm{bT}} \mathbf{F}_{\mathrm{E}},
$$

where the term $\mathbf{u}_{\mathrm{C}}=\hat{\mathbf{f}}$ generates the compensatory control signal, that approximates nonlinearities of the manipulator, contained in the function $\mathbf{f}$. The nonlinear function is approximated with a twolayer ANN [3] with tuned, fixed weights and sigmoidal bipolar neurons' activation functions. The term $\mathbf{u}_{\mathrm{PD}}=\mathbf{K}_{\mathrm{D}} \mathbf{L} \mathbf{s}$ is the form of the PD control, the term $\mathbf{v}=\mathbf{J}^{\mathrm{bT}} \mathbf{F}_{\mathrm{E}}$ can be used to offset the friction forces. Mentioned control signals influence on the quality of position control. The scheme of the 
closed loop system with control law Eq. 6 is presented in Fig. 1. The stability proof of the closedloop system is presented in $[1,3]$.

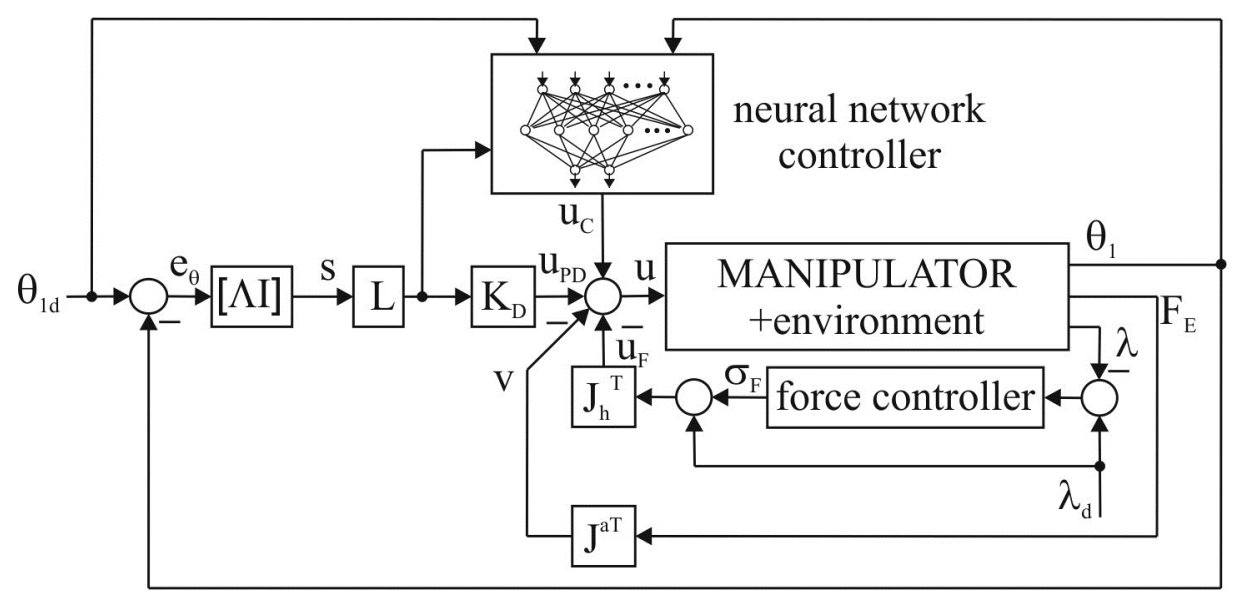

Fig. 1. The scheme of the closed-loop system

The force control is realised by the term $\mathbf{u}_{\mathrm{F}}=\mathbf{J}_{\mathrm{h}}^{\mathrm{T}}\left[\boldsymbol{\lambda}_{\mathrm{d}}+\boldsymbol{\sigma}_{\mathrm{F}}\right]$, where $\boldsymbol{\sigma}_{\mathrm{F}}$ is the control signal, which reduces force error $\tilde{\lambda}$. The choice of $\sigma_{\mathrm{F}}$ is the theme of the rest of the article.

\section{Force control}

This chapter is devoted to the aspect of the force control strategy selection. Due to the fact that the mathematical model of the robotic manipulator and the environment does not include a precise description of the contact surface features (eg. stiffness, attenuation), only relatively simple control laws can be implemented. In the presented case the $\lambda$ is a one-dimensional vector.

It was assumed that the contact surface is horizontal (lies in the xy plane) and the $\mathrm{z}$ axis is perpendicular to the surface. The surface is described by the equation $\mathrm{h}=\mathrm{z}-\mathrm{z}_{0}=0$, where $\mathrm{z}_{0}=$ const. After taking into account the manipulator kinematics and the constraint equation, jacobian $\mathbf{J}_{\mathrm{h}}$ takes the form

$$
\mathbf{J}_{\mathrm{h}}(\mathbf{q})=\left[\begin{array}{lll}
0 & 1_{2} \operatorname{cosq} & 1_{3} \operatorname{cosq}
\end{array}\right]
$$

where $\mathrm{l}_{2}, \mathrm{l}_{3}$ - link lengths, $\mathrm{q}_{2}, \mathrm{q}_{3}$ - link angles of rotation.

Conventional force control. This section presents the selected control law $\sigma_{\mathrm{F}}$. The most general approach is to use the PID controller, described by the equation

$$
\sigma_{\mathrm{F}}=\mathrm{K}_{\mathrm{FP}} \tilde{\lambda}+\mathrm{K}_{\mathrm{FD}} \dot{\tilde{\lambda}}+\mathrm{K}_{\mathrm{FI}} \int_{0}^{\mathrm{t}} \tilde{\lambda} \mathrm{dt},
$$

where $\mathrm{K}_{\mathrm{FP}}, \mathrm{K}_{\mathrm{FD}}$ and $\mathrm{K}_{\mathrm{FI}}$ denote respectively: proportional, derivative and integral gain. The proportional control $\mathrm{P}$ is frequently used in the literature $[1,3]$. Its advantage is the simplicity but the main disadvantages are a large force error lack of taking into account the dynamics of the force error signal. The insertion of the derivative term allows to take into account the force error dynamics and reduce it, but introduces a practical inconvenience, because the signal from the force sensor contains a significant measurement noise. By differentiation, into the control system is introduced a signal, which can caused so-called chatter and unstability. Therefore, it is necessary to introduce a filtration of the force error. The application of the integral gain significantly reduces the 
force error, especially in a case of the slowly changing force trajectory, but may introduces overshoot and oscillations of the interaction force.

Fuzzy force control. The conventional controllers in P, PD, PI and PID configuration were also realised using FL systems [10]. In the presented approach were used the Takagi-Sugeno FL models, with triangular affiliation functions to the fuzzy sets of premises, inference realised using PROD operator, and singleton type affiliation functions of inferences. The PID FL controller (FLC contains the rules base $R_{B}$ of $n$ rules in the form

$$
\mathrm{R}_{\mathrm{B}}^{\mathrm{n}}: \operatorname{IF}\left(\tilde{\lambda}^{*} \mathrm{IS} \tilde{\lambda} \mathrm{NB}\right) \text { AND }\left(\int \tilde{\lambda}^{*} \mathrm{dt} \text { IS i } \tilde{\lambda} \mathrm{NB}\right) \text { AND }\left(\dot{\tilde{\lambda}}^{*} \text { IS } \dot{\tilde{\lambda}} \mathrm{NB}\right) \text { THEN } \sigma_{\mathrm{F}}^{*} \text { IS } \sigma \mathrm{NVB}
$$

where $\tilde{\lambda}^{*} \in\langle-1,1\rangle$ - the normalized force error, $\dot{\tilde{\lambda}}^{*} \in\langle-1,1\rangle$ - the normalized derivative of the force error, $\int \tilde{\lambda}^{*} \mathrm{dt} \in\langle-1,1\rangle$ - the normalized integral of the force error, $\sigma_{\mathrm{F}}^{*} \in\langle-1,1\rangle$ - the normalized

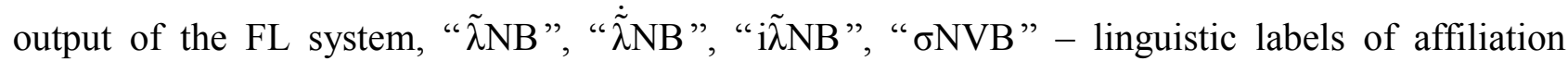
functions to the fuzzy sets of premises and inferences. On the basis of the FLC $C_{\text {PID }}$ can be realized any type of fuzzy controller (P, PD, PI), by deactivation of particular inputs to the fuzzy system. Affiliation functions to the fuzzy sets of premises and inferences, shown in Fig. 2a) for input $\tilde{\lambda}^{*}$, and in Fig. 2b) properly, can be uniformly split in space of particular inputs to the FLC. Using uniform split of input space to the fuzzy systems were realized FL controllers that meet the $\mathrm{P}$ controller $\left(\mathrm{FLC}_{\mathrm{P}}\right)$, the PI controller $\left(\mathrm{FLC}_{\mathrm{PI}}\right)$, the PD controller $\left(\mathrm{FLC}_{\mathrm{PD}}\right)$ and the PID controller ( $\left.\mathrm{FLC}_{\mathrm{PID}}\right)$. To increase quality of force control in the fuzzy PI controller were used non-uniformly split affiliation functions to premises, shown in Fig. 2c), the obtained controller is denoted as $\mathrm{FLC}_{\mathrm{N} 1}$. The second proposed controller based on $\mathrm{FLC}_{\mathrm{PI}}$, denoted as $\mathrm{FLC}_{\mathrm{N} 2}$, has non-uniformly split affiliation functions to premises and inferences (Fig. 2d).
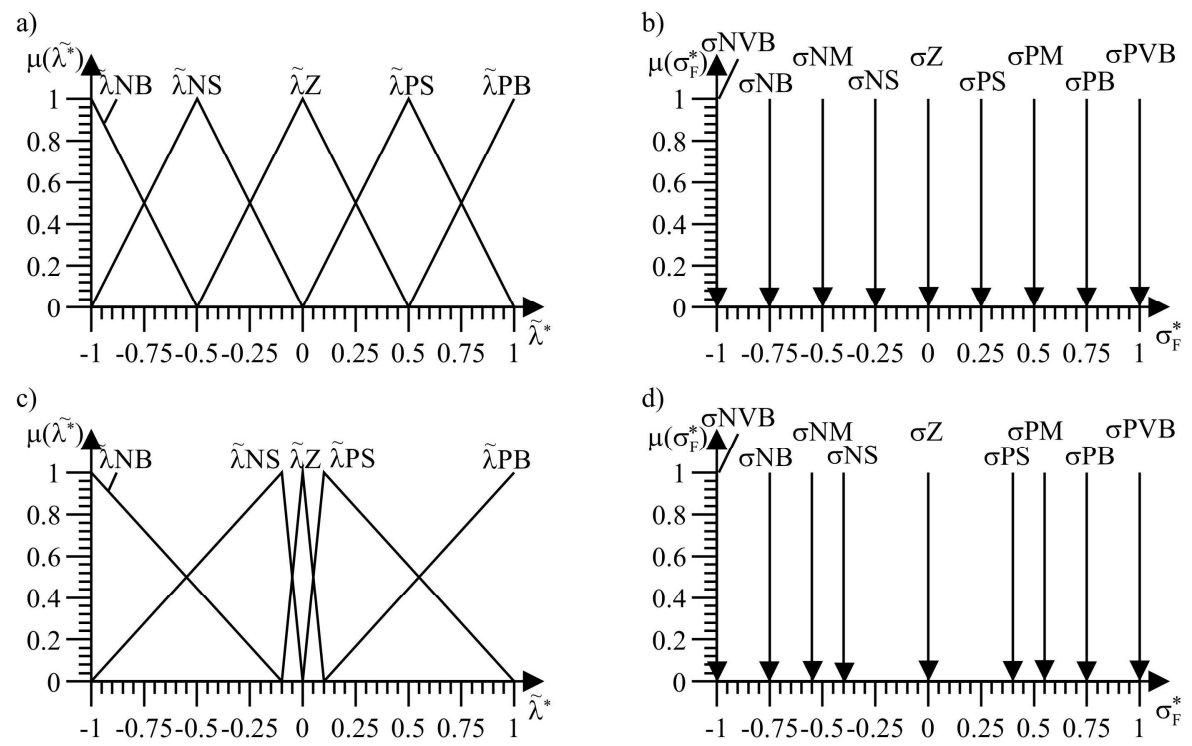

Fig. 2.a) Triangular affiliation functions to the fuzzy sets of premises for $\mathrm{FLC}_{\mathrm{PI}}$, b) singleton type affiliation functions of inferences for $\mathrm{FLC}_{\mathrm{PI}}, \mathrm{c}$ ) triangular affiliation functions to the fuzzy sets of premises for $\mathrm{FLC}_{\mathrm{N} 1}$ and $\mathrm{FLC}_{\mathrm{N} 2}$, d) singleton type affiliation functions of inferences for $\mathrm{FLC}_{\mathrm{N} 2}$

The linguistic labels of affiliations functions to the fuzzy sets denote: „NVB” - negative very big, „NB" - negative big, ..., "PS" - positive small, "PM" - positive medium, .... The FLC PID $_{\text {has }}$ three inputs and the base of $n=125$ rules, FL controllers with two inputs $\left(F C_{P D}, F L C_{P I}\right)$, have the base of $n=25$ rules, shown schematically in Fig. 3 for FLC $C_{P I}$. 


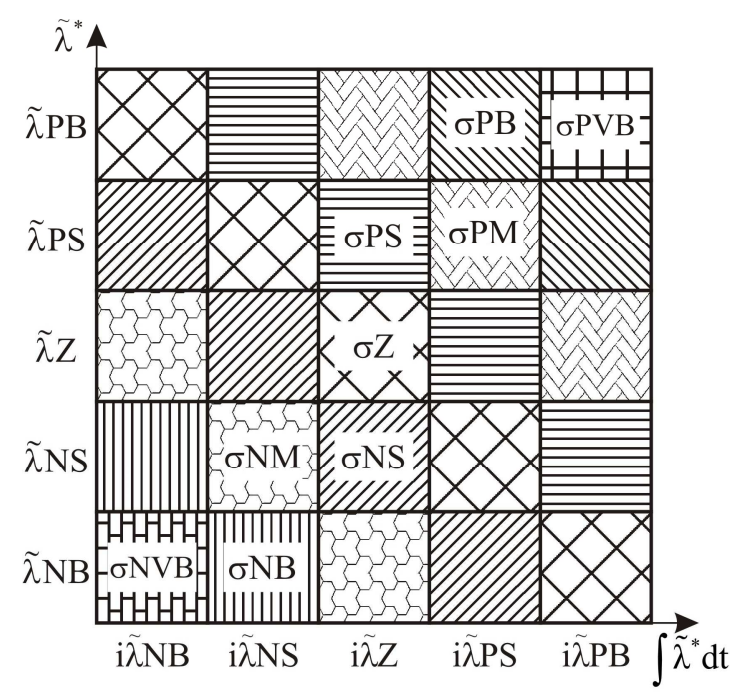

Fig. 3. The scheme of the $\mathrm{FLC}_{\mathrm{PI}}$ rules base

The FLC $_{\text {PI }}$ controller surface, shown in Fig. 4a), is a flat surface and corresponds to the PI controller surface. The change in split of affiliation functions in $\mathrm{FLC}_{\mathrm{N} 1}$ controller results in nonlinear surface of the fuzzy system shown in Fig. 4b), that consists of planes. The use of nonuniformly split fuzzy sets of premises and inferences in $\mathrm{FLC}_{\mathrm{N} 2}$, results in nonlinear surface of the fuzzy model, schematically shown in Fig. 4c).

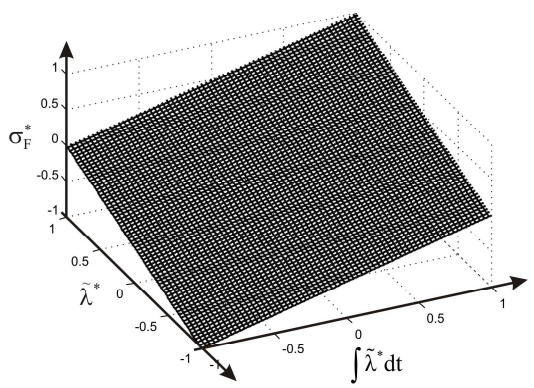

b)

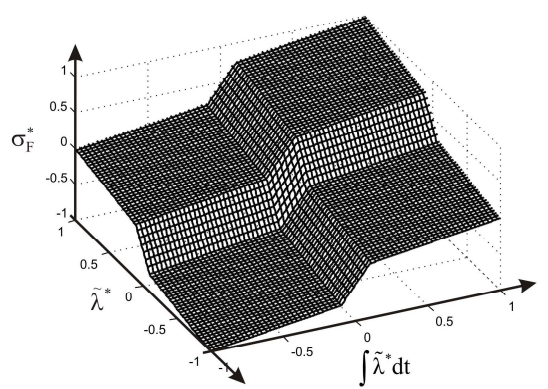

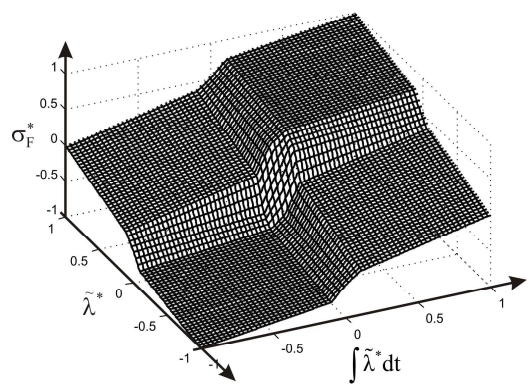

Fig. 4. The fuzzy system surface for: a) $\mathrm{FLC}_{\mathrm{PI}}$, b) fuzzy controller based on PI with non-uniformly split fuzzy sets of premises $\left(\mathrm{FLC}_{\mathrm{N} 1}\right)$, c) fuzzy controller based on PI with non-uniformly split fuzzy sets of premises and inferences $\left(\mathrm{FLC}_{\mathrm{N} 2}\right)$

The control signal of the FL controller is assumed in the form

$$
\sigma_{\mathrm{F}}=\Gamma \sigma_{\mathrm{F}}^{*},
$$

where $\Gamma$ - a positive, constant scaling rate.

\section{Experiment}

The experiment was realised using a 3 dof SCORBOT-ER 4pc robotic manipulator. Computations connected with generating of the control signals and data acquisition were realised using the dSpace DS1006 digital signal processing board. Additional robot's equipment was the force/torque sensor SCHUNK FTD-Gamma SI-130-10 mounted at the manipulator's tip and a head with a pneumatic spindle and a cutting tool, e.g. a milling cutter. The feedback necessary for the implementation of the position-force control is realised by application of optical encoders in the joints of the 
manipulator and the force/torque sensor, which measures the three components of the force and three components of the torque at the manipulator's tip.

The task of the robot was to realise the desired downforce (Fig. 5c) and the motion trajectory (Fig.5d), which results from a given path (Fig. 5a) and the velocity of the end effector (Fig. 5b).
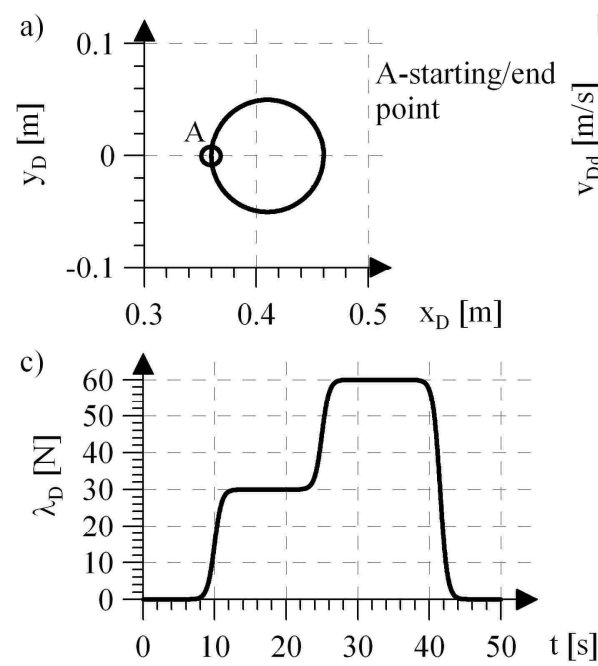
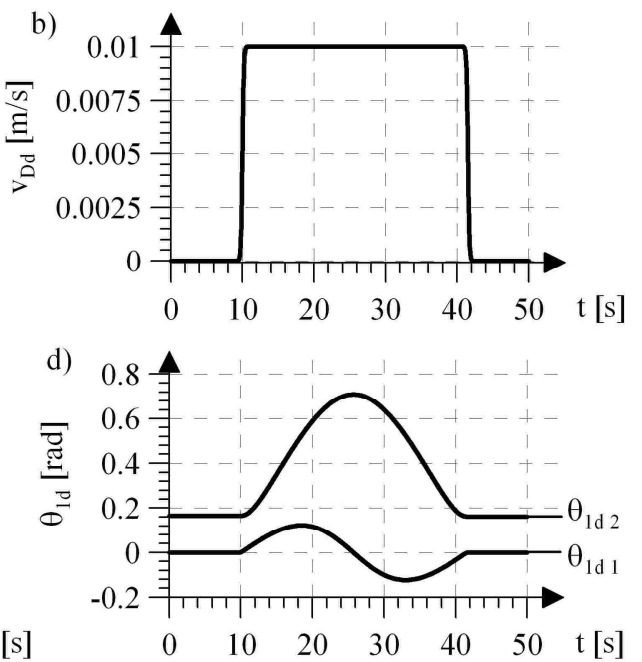

Fig. 5. a) The desired path of the tip, b) the desired motion velocity of the tip c) the desired force trajectory, d) the desired motion trajectory

During the experiments many strategies were tested, namely: conventional P, PI, PD, PID controllers and $\mathrm{FLC}_{\mathrm{P}}, \mathrm{FLC}_{\mathrm{PD}}, \mathrm{FLC}_{\mathrm{PI}}, \mathrm{FLC}_{\mathrm{PID}}, \mathrm{FLC}_{\mathrm{N} 1}, \mathrm{FLC}_{\mathrm{N} 2}$ controllers. All of the control methods were verified on the real object in order to make a comparison of control quality. The quality rating was assumed in the following form:

$$
\varepsilon_{\lambda}=\sqrt{\frac{1}{\mathrm{n}} \sum_{\mathrm{k}=1}^{\mathrm{n}} \tilde{\lambda}_{\mathrm{k}}^{2}},
$$

where $\mathrm{k}$ - the sample order number, $\mathrm{n}$ - the number of samples. In the Fig. 6 are presented force control quality ratings.

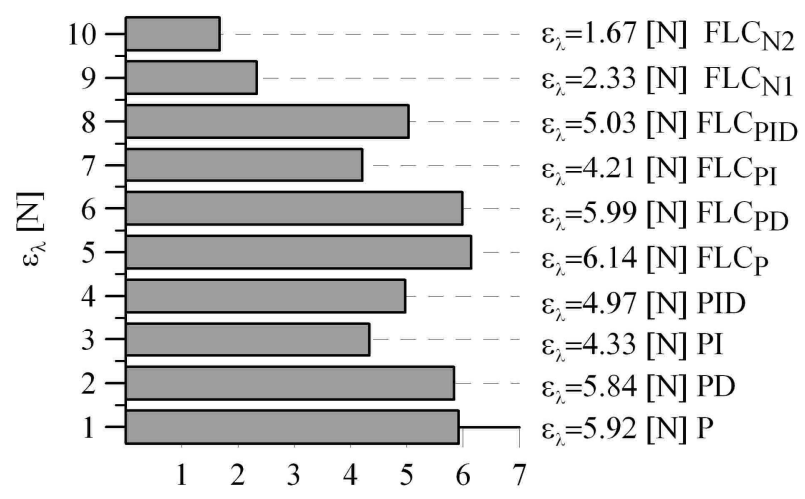

Fig. 6. Diagram of force control quality ratings

From the analysis of quality ratings follows that the best quality of the force control provides FLC $_{\mathrm{N} 2}$ controller. This is due to greater sensitivity on changes of FLC inputs in a small neighbourhood of zero, which is the most active operating area. The study also found that the application of the differentiation element to the controller does not provide a significant improvement of the control quality. This is due to the fact that differentiation of noisy force error 
signal may causes the so-called chatter, which may impair the quality of control. Therefore, the $\mathrm{K}_{\mathrm{FD}}$ gain should not be large, and so the influence of the differentiation element is not significant. In view of the study, it appears that the most appropriate regulators for the force control are regulators, whose inputs are the force error and its integral. Among these controllers the best control quality provide controllers with non-uniformly split fuzzy sets of premises and inferences.

In the Fig. 7 and Fig. 8 are presented selected results of verifications of conventional PI controller, $\mathrm{FLC}_{\mathrm{N} 1}$, and $\mathrm{FLC}_{\mathrm{N} 2}$ controllers.
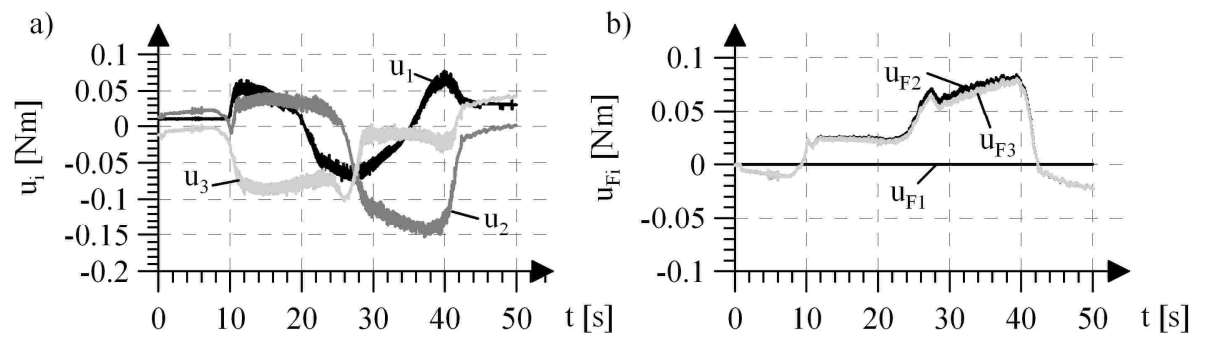

Fig. 7. Control signals of the conventional PI controller: a) the overall control signals of links 1-3, b) the force control signals for links 1-3

The Fig. 7a) shows the overall control signals generated by the hybrid controller and Fig. 7b) presents parts of the control signals generated by the force controller. A zero value of the signal $u_{\mathrm{F} 1}$ results form the fact, that the first link is not involved in control of the force. The negative control signals $\mathrm{u}_{\mathrm{F} 2}$ and $\mathrm{u}_{\mathrm{F} 3}$ in both cases at the initial phase follows that weights of links exerts the normal force on the surface but the desired force is equal zero. Afterwards the control signals increase if the desired downforce is increased. In case of other tested controllers, the control signals were similar. In Fig. 8 are presented downforces and force errors, that result from applying of the PI, FLC $\mathrm{N}_{\mathrm{N} 1}$, and FLC $_{\mathrm{N} 2}$ controllers.
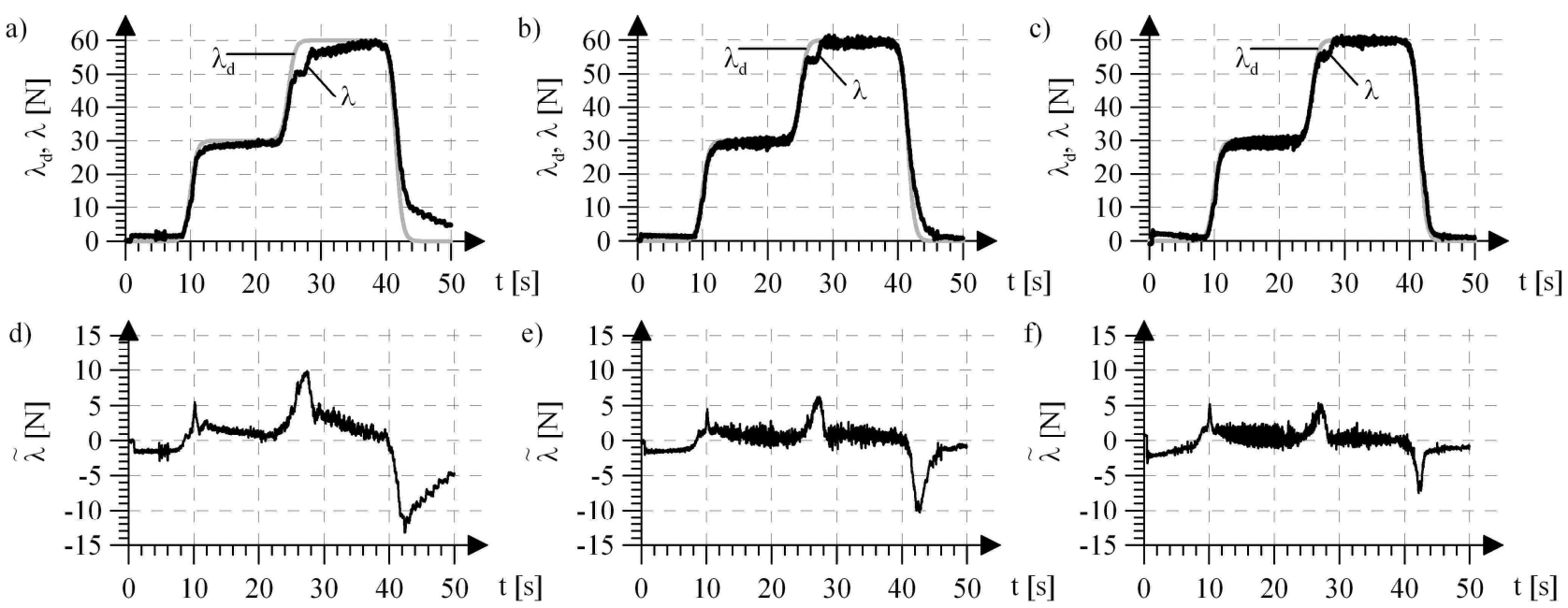

Fig. 8. Realisation of the force control: a) the desired and the realised force for conventional PI controller, $\mathrm{b}$ ) the desired and the realised force for $\mathrm{FLC}_{\mathrm{N} 1}$ controller, $\mathrm{c}$ ) the desired and the realised force for $\mathrm{FLC}_{\mathrm{N} 2}$ controller, d) the force error for conventional PI controller, e) the force error for $\mathrm{FLC}_{\mathrm{N} 1}$ controller, f) the force error for $\mathrm{FLC}_{\mathrm{N} 2}$ controller

The exerted downforce is closest to the desired force in the case of $\mathrm{FLC}_{\mathrm{N} 2}$ controller application (Fig. 8f). In other cases presented in the figure, the quality of the force control is worse. In the case of a typical literature solutions in the form of $\mathrm{P}$ controller, the quality is almost four times worse (Fig. 6) in a comparison to application of the proposed $\mathrm{FLC}_{\mathrm{N} 2}$ controller. 


\section{Summary}

The article presents comparison of conventional and fuzzy approaches to the force control of the robotic manipulator. The overall control signals consist of position control signals generated using ANN and force control signals. Few configurations of conventional controllers like P, PD, PI, PID, and corresponding to them fuzzy controllers, were tested. In order to assure better quality of the force control, the nonlinear fuzzy controller based on PI was proposed. In that controller the affiliation functions to the fuzzy sets of premises were non-uniformly split in the input space to the FLC, what resulted in a nonlinear surface of the fuzzy model and improved quality of the force control. In the second step were also applied the non-uniformly split affiliation functions to the fuzzy sets of inferences, what resulted in a lower value of quality rating, than in a case of only change in positions of the affiliation functions to the fuzzy sets of premises. The verification tests were realised using the SCORBOT-ER 4pc robotic manipulator with the six-axis force/torque sensor SCHUNK FTD-Gamma SI-130-10 and the dSpace DS1006 digital signal processing board. The researches confirmed usability of the FL systems in the force control of the robotic manipulator in repeatable machining, no attainable in a manual machining realised by a human operator.

\section{Acknowledgement}

This paper is supported by Polish Government under Contract INNOTECHK2/IN2/66/182991/NCBR/13.

\section{References}

[1] F.L. Lewis, S. Jagannathan, A. Yesildirek, Control of Robot Manipulators and Nonlinear Systems, Taylor \& Francis, London, 1999.

[2] A. Šabanović, K. Ohnishi, Motion control system, IEEE Press, Singapore, 2011.

[3] P. Gierlak, Hybrid Position/Force Control of the SCORBOT-ER 4pc Manipulator with Neural Compensation of Nonlinearities, Lecture Notes in Comput. Sci. 7268 (2012) 433-441

[4] P. Gierlak, W. Żylski, Adaptive hybrid position/force control of manipulator, Int. J. of Applied Mechanics and Engineering 17, No. 3 (2012) 811-825

[5] N. Kumar, V. Panwar, N. Sukavanam, S.P. Sharma, J.-H. Borm, Neural Network Based Hybrid Force/Position Control for Robot Manipulators, Int. J. Precis. Eng. Manuf. 12, No. 3 (2011) 419426

[6] F. Yu, M. Minami, T. Maeba, A. Yanou, Constraint-combined Force/Position Hybrid Control Method with Lyapunov Stability, Proc. SICE Annual Conference (2011) 671-676

[7] P. Gierlak, W. Żylski, Tracking control of manipulator, Methods and Models in Automation and Robotics 14, part 1 (2009) 623-628

[8] N. Mendes, P. Neto, J.N. Pires, P. Moreira, Fuzzy-PI Force Control for Industrial Robotics, Commun. in Computer and Info. Sci. 103 (2010) 322-329

[9] K. Tchoń, A. Mazur, I. Dulęba, R. Hossa, R. Muszyński, Manipulators and mobile robots: models, movement planning and control, AOW PLJ, Warsaw, 2000 (in Polish).

[10]A. Piegat, Fuzzy modelling and control, Exit, Warsaw, 2003 (in Polish). 